\title{
Comparative transcriptomic analysis reveals female-biased olfactory genes potentially involved in plant volatile-mediated oviposition behavior of Bactrocera dorsalis
}

Li Xu $u^{1,2}$, Kai-Yue Tang ${ }^{1,2}$, Xiao-Feng Chen ${ }^{1,2}$, Yong Tao ${ }^{1,2}$, Hong-Bo Jiang ${ }^{1,2}$ and Jin-Jun Wang ${ }^{1,2^{*}}$ (D)

\begin{abstract}
Background: Olfactory systems take on important tasks to distinguish salient information from a complex olfactory environment, such as locating hosts, mating, aggression, selecting oviposition sites, and avoiding predators. The olfactory system of an adult insect consists of two pairs of main olfactory appendages on the head, the antennae, and the palps, which are covered with sensilla. Benzothiazole and 1-octen-3-ol could elicit oviposition behavior in gravid B. dorsalis are regarded as oviposition stimulants. However, the mechanism for how B. dorsalis percepts benzothiazole and 1-octen-3-ol still remains unknown.
\end{abstract}

Results: We conducted a comparative analysis of the antennal transcriptomes in different genders of $B$. dorsalis using Illumina RNA sequencing (RNA-seq). We identified a total of 1571 differentially expressed genes (DEGs) among the two sexes, including 450 female-biased genes and 1121 male-biased genes. Among these DEGs, we screened out 24 olfaction-related genes and validated them by qRT-PCR. The expression patterns of these genes in different body parts were further determined. In addition, we detected the expression profiles of the screened female-biased chemosensory genes in virgin and mated female flies. Furthermore, the oviposition stimulantsinduced expression profilings were used to identify chemosensory genes potentially responsible for benzothiazole and 1-octen-3-ol perception in this fly.

Conclusions: The results from this study provided fundamental data of chemosensory DEGs in the B. dorsalis antenna. The odorant exposure assays we employed lay a solid foundation for the further research regarding the molecular mechanism of benzothiazole and 1-octen-3-ol mediated oviposition behavior in B. dorsalis.

Keywords: Bactrocera dorsalis, Antenna, Chemosensory, Benzothiazole, 1-octen-3-ol

\footnotetext{
* Correspondence: wangjinjun@swu.edu.cn

'Key Laboratory of Entomology and Pest Control Engineering, College of Plant Protection, Southwest University, Chongqing 400715, China

${ }^{2}$ State Cultivation Base of Crop Stress Biology for Southern Mountainous Land, Academy of Agricultural Sciences, Southwest University, Chongqing 400715, China
}

(c) The Author(s). 2021 Open Access This article is licensed under a Creative Commons Attribution 4.0 International License, which permits use, sharing, adaptation, distribution and reproduction in any medium or format, as long as you give appropriate credit to the original author(s) and the source, provide a link to the Creative Commons licence, and indicate if changes were made. The images or other third party material in this article are included in the article's Creative Commons licence, unless indicated otherwise in a credit line to the material. If material is not included in the article's Creative Commons licence and your intended use is not permitted by statutory regulation or exceeds the permitted use, you will need to obtain permission directly from the copyright holder. To view a copy of this licence, visit http://creativecommons.org/licenses/by/4.0/. The Creative Commons Public Domain Dedication waiver (http://creativecommons.org/publicdomain/zero/1.0/) applies to the data made available in this article, unless otherwise stated in a credit line to the data. 


\section{Background}

The oriental fruit fly, Bactrocera dorsalis (Hendel), is a destructive agricultural pest that is widely distributed in many areas of the Asian countries [1]. It causes severe economic loss and trade restrictions to vegetables and fruits by ovipositing inside more than 450 host plant species (USDA, 2016). Recently, owing to its strong environmental adaptiveness, invasive ability [2], and the quick development of resistance [3], the damage produced by this pest has become increasingly serious. Therefore, reducing damage by $B$. dorsalis is becoming a focus of research efforts worldwide. Currently, the most economical and effective way to control $B$. dorsalis is olfaction-based trapping [4], which indicates that the olfaction system plays an important role in management of this pest.

Olfactory systems take on important tasks to distinguish salient information from a complex olfactory environment [5]. It is reported that the exceptional sensing ability plays essential roles in locating hosts, mating, aggression, selecting oviposition sites, choosing habitats, and avoiding predators [6]. The olfactory system of an adult insect consists of two pairs of main olfactory appendages on the head, the antennae, and the palps [7], which are covered with sensilla. The peripheral olfactory sensing of insects mainly relies on the olfactory sensory neurons (OSNs), which are located at the olfactory sensilla of the antenna. Recently, RNA sequencing and bioinformatics-based approaches have been rapidly developed to analyze insect communication systems [8]. So far, many antennal transcriptome projects from various insect species have been completed, such as Helicoverpa armigera [9], Chilo suppressalis [10], Cotesia vestalis [11] and Ostrinia furnacalis [12]. It has demonstrated that chemoreception involves several families of proteins including odorant binding proteins (OBPs), sensory neuron membrane proteins (SNMPs), chemosensory proteins (CSPs), odorant degrading enzymes (ODEs), odorant receptors (ORs), gustatory receptors (GRs), and ionotropic receptors (IRs) [6].

Phytochemicals from host plants are known to affect sexual behaviors and physiology in a wide range of insects. In several phytophagous insect species, males are attracted to plant volatile compounds to increase the possibility of encountering females [13]. In $B$. dorsalis, it is well known that methyl eugenol (ME) is strongly attractive to males [14]. Based on the high potency and sex pheromone-like behavior of ME, trapping systems have been developed to manage $B$. dorsalis and as well as additional molecular mechanistic analysis of $B$. dorsalis-ME interactions $[15,16]$, but primarily with male flies. However, the oviposition by females is the main mechanism of fruit damage by insects. Therefore, it is important and necessary to develop new methods for trapping females and discovering oviposition stimulants may have greater potential in trapping gravid females [17].

Suitable oviposition sites are crucial for female insects due to their central role in successful development of offspring. During the oviposition, the olfactory cues from the external environment are important for female insects to make an appropriate choice for where to lay eggs. Previous studies showed that benzothiazole and 1octen-3-ol could elicit oviposition behavior in gravid $B$. dorsalis and they are regarded as oviposition stimulants [17, 18]. The identification of oviposition stimulants would provide clues for understanding the co-evolution mechanism of $B$. dorsalis and their host plants, which could provide better methods for trapping female flies. However, the mechanism for how $B$. dorsalis percepts benzothiazole and 1-octen-3-ol still remains unknown. Previous research on oviposition stimulants were mainly focused on lepidopteran insects [17] and few studies have been reported in $B$. dorsalis. To better understand the mechanism of $B$. dorsalis perception of reported oviposition stimulants benzothiazole and 1-octen-3-ol, we sequenced and analyzed the antennal transcriptomes in both sexes of $B$. dorsalis with Illumina RNA sequencing (RNA-seq). We screened out and confirmed chemosensory DEGs, and female-biased chemosensory genes were selected to detect their expression profiles in virgin and mated female flies. In addition, we measured the expression levels of the female-biased chemosensory genes after exposed virgin and mated female flies to benzothiazole and 1-octen-3-ol for $2 \mathrm{~h}$ to determine chemosensory genes which may be responsible for oviposition stimulation. The evidence presented here provides fundamental data for male- and female-biased chemosensory genes and establishes an odorant exposure assay to lay a solid foundation for the further research into the molecular mechanisms of benzothiazole- and 1-octen-3-ol-mediated oviposition behavior in B. dorsalis.

\section{Results}

\section{Transcriptome sequencing and assembly}

A library of the male and female antenna was developed by Illumina sequencing with three replicates, which generated about $37.57 \mathrm{~Gb}$ of raw data. Each library was of good quality with Q20 and Q30 percentage of over 97.86 and $94.41 \%$, respectively. After initial adaptor trimming and quality filtering, $3.72 \times 10^{8}$ clean reads were obtained for sequencing from the six samples (Table 1). There were $8.67 \times 10^{5}$ contigs with an N50 length of $425 \mathrm{bp}$ assembled from clean data. The contigs were further assembled into 58,951 unigenes with a mean length of $774 \mathrm{bp}$ using the paired-end joining and gap-filling method (Table 2). Among the total unigenes, the lengths of 48,337 unigenes (82\%) were between 200 bp to 1000 
Table 1 Statistical sequencing data of the RNA-Seq reads for the examined samples

\begin{tabular}{|c|c|c|c|c|c|c|}
\hline \multirow[t]{2}{*}{ Group Name } & \multicolumn{3}{|l|}{ Female } & \multicolumn{3}{|l|}{ Male } \\
\hline & FAn1 & FAn2 & FAn3 & MAn1 & MAn2 & MAn3 \\
\hline No. clean reads & $5.99 \times 10^{7}$ & $5.64 \times 10^{7}$ & $6.04 \times 10^{7}$ & $6.50 \times 10^{7}$ & $6.97 \times 10^{7}$ & $6.01 \times 10^{7}$ \\
\hline No. clean bases & $8.98 \times 10^{9}$ & $8.46 \times 10^{9}$ & $9.07 \times 10^{9}$ & $9.74 \times 10^{9}$ & $10.46 \times 10^{9}$ & $9.01 \times 10^{9}$ \\
\hline GC content (\%) & $39.05 \%$ & $38.23 \%$ & $38.16 \%$ & $37.89 \%$ & $38.49 \%$ & $38.43 \%$ \\
\hline Q20 (\%) & $97.93 \%$ & $97.86 \%$ & $97.87 \%$ & $97.91 \%$ & $97.96 \%$ & $97.95 \%$ \\
\hline Q30 (\%) & $94.48 \%$ & $94.41 \%$ & $94.41 \%$ & $94.53 \%$ & $94.61 \%$ & $94.58 \%$ \\
\hline
\end{tabular}

FAn female antenna, MAn male antenna; 1, 2, 3 represent different biological replicates respectively. Clean reads, the filtered sequencing data; GC content, the percentage of the number of $\mathrm{G}$ and $\mathrm{C}$ in the total base; Q20 and Q30 refers to the error probability given to the identified base during the base calling process of the sequencing process

bp, 10,614 unigenes (12\%) were over $1000 \mathrm{bp}$, and no unigene was less than $200 \mathrm{bp}$.

\section{Annotation of predicted transcripts}

Unigene sequences were annotated using BLASTX against the KOG, Swissprot, TrEML, GO, and nonredundant (NR) NCBI protein databases. All annotation details were obtained from the combined genes with similarities $>30 \%$. In all, a total of 32,992 (55.97\%) assembled unigene sequences showed annotation hits at least one of the protein or nucleotide databases and 5003 unigenes (8.5\%) showed annotation hits in all databases. Among the annotations in these databases, the best hits were in the NR and TrEML databases where the number of annotated genes were 31,045 (52.66\%) and 31,501 (53.44\%), respectively (Table 2). According to the NR database, more than half of annotated unigenes (16,642 unigenes, 53.6\%) showed homology with the oriental fruit fly, B. dorsalis (Figure S1), whereas there were 3782 unigenes (12.2\%) matched to other species. A four-way Venn diagram plot (Figure S2) also showed the assembled unigenes annotated against NR,

Table 2 Statistics of the assembled sequences

\begin{tabular}{ll}
\hline Group Name & Number \\
\hline Total number of clean reads & $3.72 \times 10^{8}$ \\
Total number of contigs & $8.67 \times 10^{5}$ \\
Total assembled bases & $5.57 \times 10^{10}$ \\
Total number of unigenes & 58,951 \\
Unigene N50 (bp) & 1276 \\
Maximum unigene length (bp) & 19,935 \\
Minimum unigene length (bp) & 201 \\
Average unigene length (bp) & 774 \\
Unigenes annotation in NR & 31,045 \\
Unigenes annotation in Swiss-Prot & 20,777 \\
Unigenes annotation in KEGG & 7388 \\
Unigenes annotation in GO & 19,751 \\
Unigenes annotation in KOG & 16,103 \\
\hline
\end{tabular}

KEGG, Swissprot, and KOG databases. This diagram indicated that 6323 unigenes were annotated by all four databases, whereas 8806 unigenes had homologous sequences both in NR, Swissprot, and KOG databases.

\section{Analysis of DEGs}

DEG analysis was performed to gain the antennal transcriptome differences between the male and female adults. All of the DEGs were compared by normalizing the number of unambiguous tags in each library to RPKM (reads per kilobase mapped). To verify the reliability of the samples, the correlation between each sample was conducted by Pearson's correlation analysis (Figure S3). The correlation coefficients range from 0.94 to 1 , indicating the reliability of samples. Compared with the female group, the expression levels of 1571 unigenes were significantly different in the male group (Fig. 1). We identified 450 female-biased genes and 1121 malebiased genes from these DEGs. The fold change $\left(\log _{2}\right.$ ratio) was calculated using the mean expression level of genes in male antenna divided to that in female antenna. Among DEGs, 24 genes were associated with olfaction including 10 OBPs, 12 ORs, 1 SNMP, and 1 GR. Their annotation information and fold changes are shown in Table 3.

\section{qRT-PCR validation}

To confirm the DEG results from Illumina sequencing, we confirmed the 24 chemosensory genes by qRT-PCR (Fig. 2). Among them, 12 genes were highly expressed in the female antennae and the other 12 genes were highly expressed in the male antennae. As expected, the results of the qRT-PCR were basically consistent with the RNASeq data. All candidate chemosensory genes had similar expression change trends as the DEG analysis from Illumina sequencing. However, there were some genes whose fold changes were different between DEG analysis and $\mathrm{qPCR}$ results. For instance, a 9.28-fold change in DEG analysis versus about 3.3-fold in the result of qPCR for GR 2a, a 6-fold change in DEG analysis versus about 2.4-fold in qPCR detection for OBP 5, and 12.14-fold 


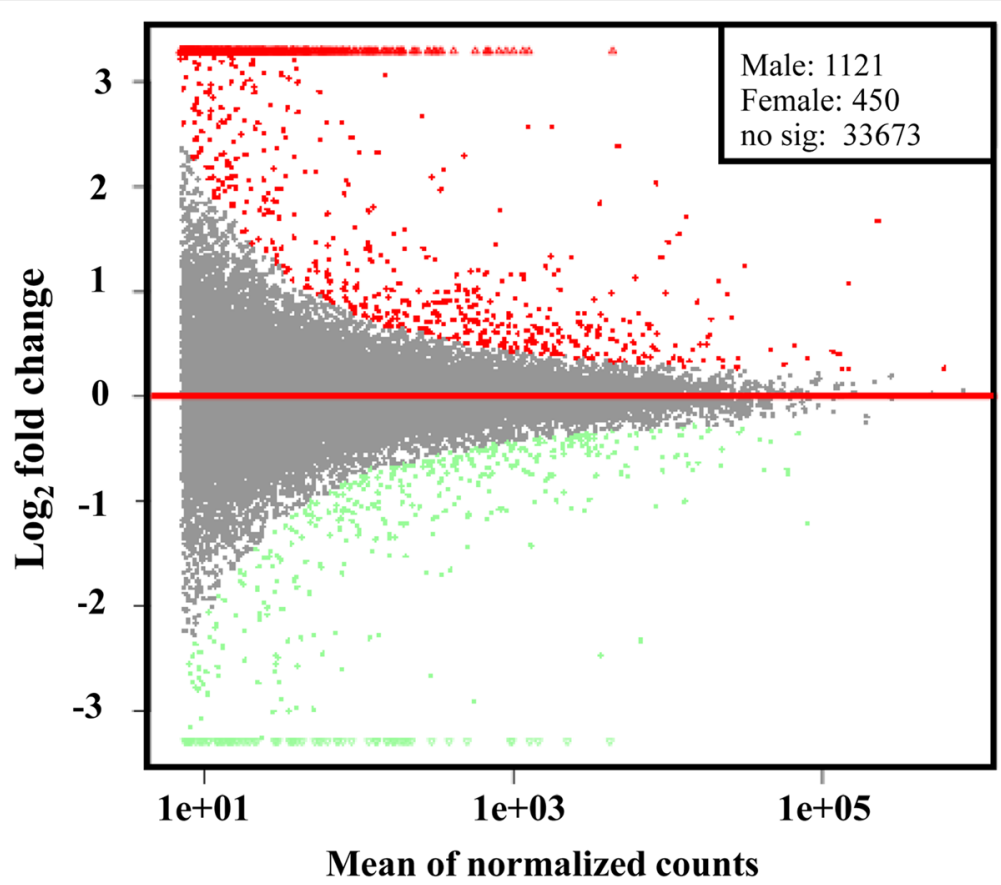

Fig. 1 The DEG plot of FAn vs. MAn with $P$-value $<0.05$. The $X$-axis indicates the mean of the normalized sequence count between the two groups, and the $Y$-axis represents the fold-change of gene expression levels ( $\log _{2}$-fold change). The genes above the red line with red points are more highly expressed in the male adults and those below the red line with green points are more highly expressed in female adults. The grey points were the genes with no significant differences. The legend on the upper right shows the specific number of genes highly expressed in males and females. Abbreviations: FAn, female antenna; MAn, male antenna

change in DEG analysis versus about 2-fold in the result of qPCR for OBP 99a. The largest difference in fold change between DEG analysis and $\mathrm{qPCR}$ validation was OBP c21, which showed 234-fold change in DEG analysis and 4.6-fold in qPCR validation. Meanwhile, the 24 selected genes showed a significant correlation $\left(R^{2}=\right.$ $0.90, P<0.01$, Spearman correlation coefficient) between RNA-seq and qPCR results (Figure S4). It indicated the reliable of RNA-seq data in this study.

In addition, the expression patterns of these 24 candidate genes were further investigated in different body parts of B. dorsalis across eight kinds of tissue (Fig. 3). As indicated by the qRT-PCR, all candidate genes were highly expressed in tissues that are rich in sensilla like antenna, proboscis, and maxillary palps, except for OBP 56d-1. OBP 56d-1 expression was highest in abdomen cuticles.

To investigate the expression profile of the 12 femalebiased genes in virgin and mated flies, the qPCR detection was performed with 15-day-old virgin and mated flies, respectively (Fig. 4). The expression level of five candidate genes was significantly upregulated after mating including SNMP 1, OBP 56d-1, OBP 56d-2, OR 7a2 , and OR 43b-2. In contrast, the expression levels of OBP 3 and OR 7a-1 decreased significantly after mating. Expression levels of the other five candidate genes showed no significant differences between virgin and mated flies.

\section{Transcript level detection of female-biased DEGs after odorant exposure}

To confirm the characteristic of oviposition stimulants benzothiazole and 1-octen-3-ol for female flies, the transcript levels of the screened 12 female-biased genes were measured after exposing virgin and mated flies to benzothiazole and 1-octen-3-ol for $2 \mathrm{~h}$. The results showed that after exposure to benzothiazole for $2 \mathrm{~h}$, the expression level of OBP 56d-2 increased significantly in virgin flies and was about 1.58-fold changed from MO exposure (Fig. 5). In mated flies, the expression level of OBP 56d-2 increased significantly as well after exposure to benzothiazole (Fig. 5). However, the fold change was much higher, about a 5-fold change. As for 1-octen-3-ol exposure, the expression level of OBP 56d-1, OBP 56d2, and OR 7a-2 increased in virgin flies (Fig. 6). For mated flies, the expression level of these three genes increased as well, and the fold changes were much higher (Fig. 6). In addition, except for the three changed genes in virgin female flies, the expression level of OR 43b-2 increased when exposed mated females to 1-octen-3-ol. However, the fold change was lower the other three genes.

\section{Discussion}

In this study, the sequencing quality was reliable according to our evaluation by the Illumina's base-caller 
Table 3 List of candidate olfactory genes from comparative antennal transcriptome

\begin{tabular}{|c|c|c|c|c|c|c|}
\hline ID & FAn & MAn & $\log _{2}$ fold change & $P$ adj & Gene description & Abbreviation \\
\hline DN122377_c0_g1_i1 & 0.010 & 0.090 & 3.21 & 0.021 & putative gustatory receptor $2 \mathrm{a}$ & GR 2a \\
\hline DN154971_c8_g8_i2 & 424.03 & 278.82 & -0.56 & 0.0023 & sensory neuron membrane protein 1 -like & SNMP 1 \\
\hline DN130398_c1_g1_i1 & 295.53 & 143.73 & -1.00 & $2.72 \times 10^{-9}$ & odorant binding protein 3 & OBP 3 \\
\hline DN120332_C0_g1_i1 & 1.04 & 6.21 & 2.68 & $1.33 \times 10^{-16}$ & odorant binding protein 3 & OBP 5 \\
\hline DN135531_c41_g6_i1 & 3502.75 & 4547.85 & 0.42 & 0.00067 & odorant binding protein 19d & OBP 19d \\
\hline DN137330_c11_g9_i1 & 2987.73 & 3827.46 & 0.40 & 0.0015 & odorant-binding protein 22 & OBP 22 \\
\hline DN71860_c0_g1_i1 & 7.19 & 3.79 & -0.86 & 0.046 & odorant-binding protein 56d-like & OBP 56d-1 \\
\hline DN103452_C0_g1_i1 & 308.56 & 173.67 & -0.77 & $4.78 \times 10^{-6}$ & odorant-binding protein 56d-like & OBP $56 \mathrm{~d}-2$ \\
\hline DN141472_c1_g1_i1 & 0.31 & 1.58 & 2.47 & $7.77 \times 10^{-9}$ & odorant-binding protein 57c & OBP 57c \\
\hline DN117742_C0_g1_i1 & 0.36 & 4.33 & 3.60 & $2.69 \times 10^{-21}$ & odorant-binding protein 99a-like & OBP 99a \\
\hline DN84733_c0_g1_i1 & 0.26 & 1.78 & 2.78 & $8.56 \times 10^{-5}$ & odorant binding protein c11 & OBP c11 \\
\hline DN102208_C0_g1_i1 & 0.017 & 4.13 & 7.88 & $1.95 \times 10^{-15}$ & odorant binding protein c21 & OBP c21 \\
\hline DN125498_C0_g1_i1 & 5.23 & 2.22 & -1.19 & 0.0036 & odorant receptor 7a-like & OR 7a-1 \\
\hline DN149599_c2_g17_i1 & 12.42 & 8.55 & -0.52 & 0.0089 & odorant receptor 7a-like & OR 7a-2 \\
\hline DN153156_c12_g20_i1 & 46.83 & 122.17 & 1.45 & $1.59 \times 10^{-9}$ & odorant receptor 7a-like & OR 7a-3 \\
\hline DN153156_c12_g2_i1 & 8.90 & 21.03 & 1.31 & 0.00041 & odorant receptor 43b-like & OR 43b-1 \\
\hline DN136265_c1_g6_i2 & 34.73 & 2.02 & -4.08 & $4.40 \times 10^{-19}$ & odorant receptor 43b-like & OR 43b-2 \\
\hline DN153156_c12_g35_i1 & 14.80 & 61.08 & 2.09 & $6.15 \times 10^{-15}$ & odorant receptor 43b-like & OR 43b-3 \\
\hline DN154906_c6_94_i1 & 8.34 & 4.90 & -0.72 & 0.041 & odorant receptor 43b-like & OR 43b-4 \\
\hline DN77796_c0_g4_i1 & 4.93 & 1.16 & -2.031 & 0.0012 & orant receptor 43b-like & OR 43b-5 \\
\hline DN151752_c1_g26_i2 & 5.48 & 3.40 & -0.67 & 0.015 & odorant receptor 67d-like & OR 67d-1 \\
\hline DN151752_c1_g22_i1 & 38.69 & 22.02 & -0.78 & 0.040 & odorant receptor 67d-like & OR $67 d-2$ \\
\hline DN135169_c4_g1_i1 & 11.41 & 8.66 & -0.38 & 0.033 & odorant receptor 74a-like & OR 74a \\
\hline DN125969_C0_g1_i1 & 0.014 & 0.16 & 3.61 & 0.0006 & odorant receptor 94a-like & OR 94a \\
\hline
\end{tabular}

ID the gene ID, FAn female antenna, MAn male antenna. The values of FAn and MAn are the average expression levels of three biological replications; the fold change value was obtained by calculating the shrinkage model in the differential analysis software (DESeq2) and fold change represents the fold change of expression level from females to males; $P$ adj, the corrected $P$-value; Gene description, the description of gene function

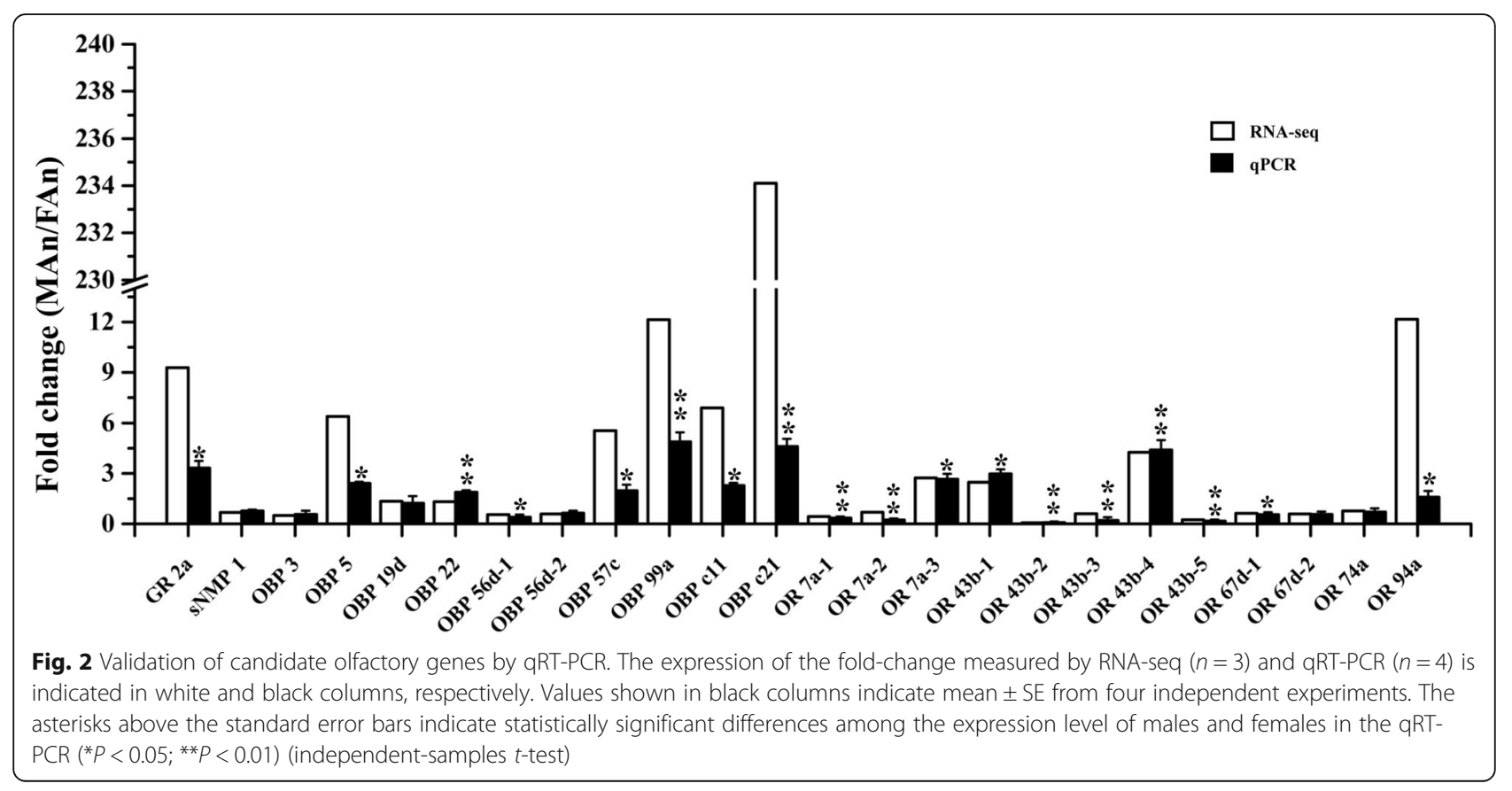




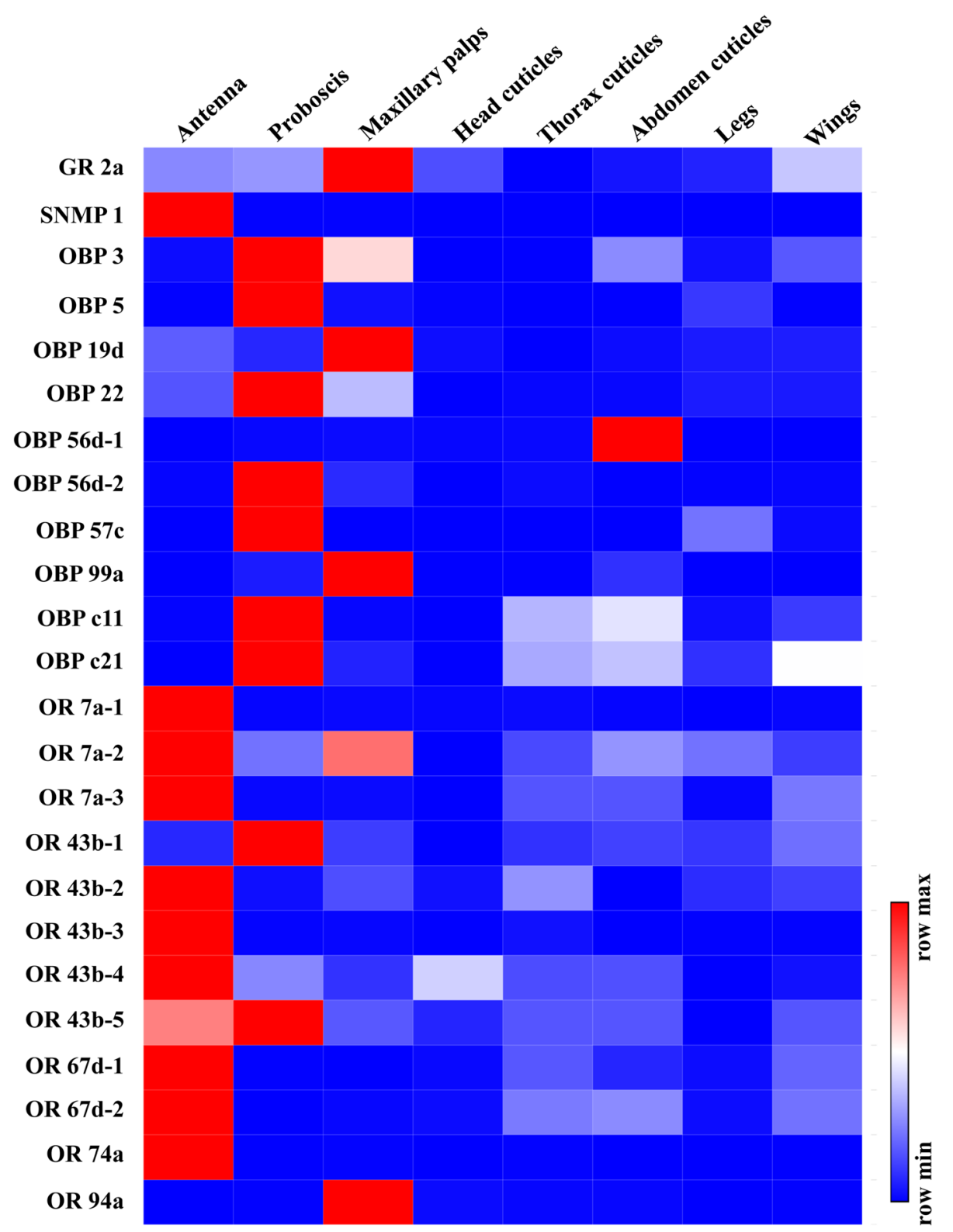

Fig. 3 The expression patterns of candidate olfactory genes in different body segments. Body segments from female flies included eight parts: antenna, proboscis, maxillary palps, head cuticles, thorax cuticles, abdomen cuticles, legs, and wings. The heatmap was generated using the online website https://software.broadinstitute.org/morpheus/. Each row represents a gene, and each column represents a tissue. A relative color scheme used the minimum and maximum values in each row to convert values to the intensity of colors. Red represents the relative higher expression level, and blue is the relative lower expression level

Bustard software. More than $94.4 \%$ of clean reads had quality scores higher than the Q30 level (an error probability of $0.1 \%$ ) (Table 1 ). Compared with our previous study [19], we have substantially expanded our dataset of chemosensory DEGs in different sexes of $B$. dorsalis antenna.

Sexual dimorphisms in olfaction-mediated behaviors have drawn extensive attention both in terms of understanding insect behavior and as potential mechanisms for insect pest control. However, it remains still unknown whether males and females have the same ability of chemoperception. DEG analysis generated from the comparative transcriptomics was proven to be a powerful tool to explore sex-biased genes involved in the sexual dimorphisms of insect behaviors [9, 20, 21]. Insects perceive the surrounding through different organs [22], among which antennae are the most important and play a central role in the life of the insect. Therefore, antennae were used to conduct comparative transcriptomic analysis between both sexes in this study. In the present 


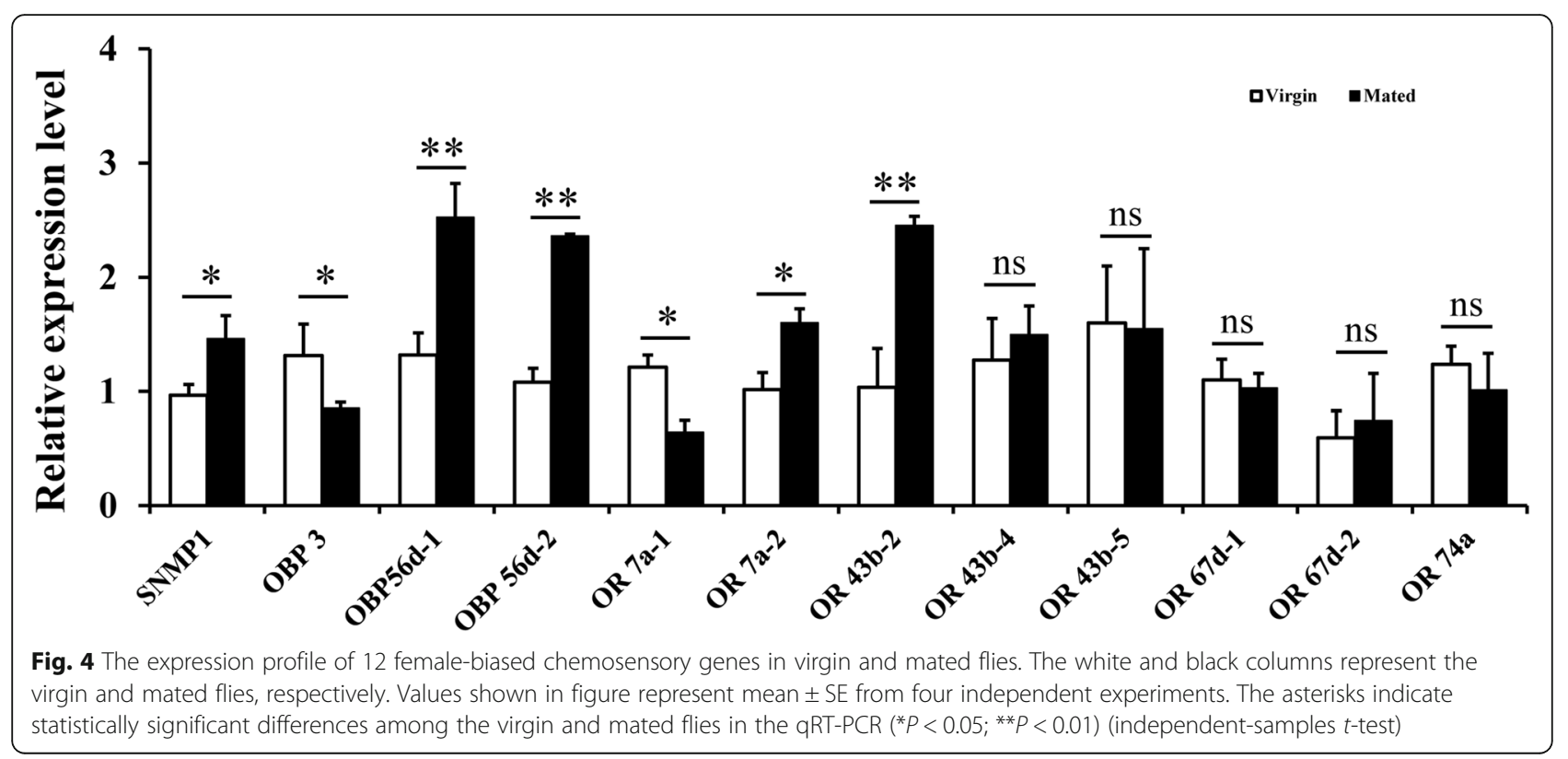

study, we screened out 24 chemosensory DEGs, it was similar to a previous study which identified 27 sexbiased chemosensory genes in Drosophila suzukii antennae [23].

All of the candidate chemosensory genes detected by qRT-PCR showed similar change trends as indicated by DEG analysis, suggesting that a DEG approach can be a powerful way to screen out candidate genes in a highthroughput manner. However, there were some foldchange magnitude differences between qRT-PCR and DEG analysis. The reason may be due to sensitivity bias between two methods or different statistical approaches and thresholds in qRT-PCR and DEG analysis [24]. In addition, we also examined the expression profiles of these chemosensory genes in different appendages in female adults. As indicated by the results, with exception to OBP 56d-1, all candidate genes were high expressed in tissues rich in sensilla like antenna, proboscis, and maxillary palps, suggesting these genes may play an important role in olfaction or gustation. However, the expression of OBP 56d-1 was highest in abdomen cuticles. Therefore, we inferred that abdomen cuticles may contain many sensilla, especially on the ovipositor. A recent study showed that HassOR31 highly expressed in

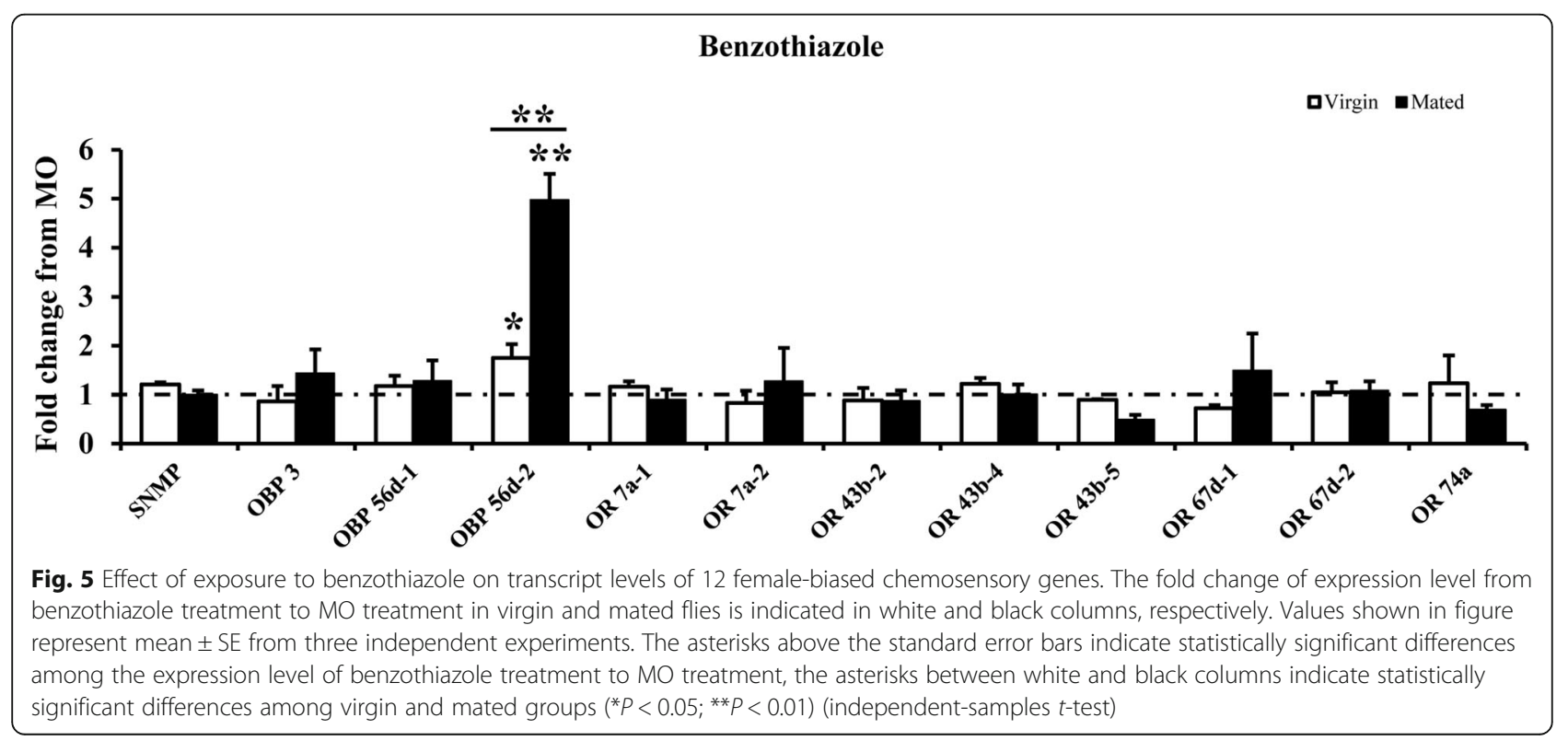




\section{1-octen-3-ol}

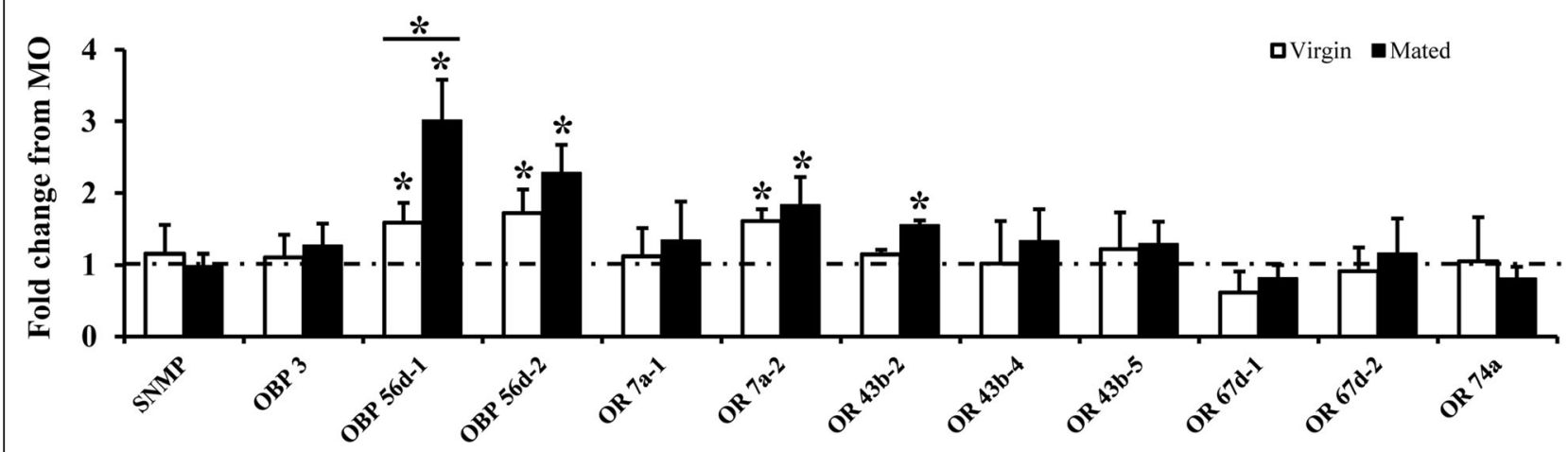

Fig. 6 Effect of exposure to 1-octen-3-ol on transcript levels of 12 female-biased chemosensory genes. The fold change of expression level from 1-octen-3-ol treatment to MO treatment in virgin and mated flies is indicated in white and black columns, respectively. Values shown in figure represent mean \pm SE from three independent experiments. The asterisks above the standard error bars indicate statistically significant differences among the expression levels of 1-octen-3-ol treatment to $\mathrm{MO}$ treatment, the asterisks between white and black columns indicate statistically significant differences among virgin and mated group ( ${ }^{*} P<0.05 ;{ }^{*} P<0.01$ ) (independent-samples t-test)

ovipositor was involved in detecting host-plant volatiles for determining precise oviposition sites [25]. This gene may exert a function associated with host-plant volatile detection or stimulation of egg lay.

Olfactory cues influence insects in many different ways [26]. For instance, oviposition is one of the most important female behaviors and has great influence on the fitness of insect species. Since egg distribution is determined by adult females, searching for suitable oviposition sites plays an important role in oviposition behaviors for gravid females [27]. In addition, oviposition stimulants could enhance fecundity of the gravid females [18]. Thus, whether these sexually dimorphic behaviors are associated with the sex-biased expression of chemosensory genes needs to be clarified. Previous studies indicated that several pheromone receptors, which are specifically expressed in male antenna, are responsible for the male attraction by sex pheromones produced by females in Heliothis virescens [28]. In Maruca vitrata, MvitGOBP1-2 showed the highest expression levels in female antennae, and it was reported to bind with some floral volatiles, which may effectively attract female moths [29]. In addition, dsRNA mediated knock down of the male-biased genes OBP 99a dramatically reduced courtship behavior in $B$. dorsalis males [30]. Based on the previous studies, we speculated that the chemosensory genes responsible for special odorants, which could elicit sexual behaviors, may have sex-biased expression. Therefore, the differentially expressed chemosensory genes presented in this study should be involved in some sexual behaviors.

It has been reported that benzothiazole and 1-octen-3ol serve as oviposition stimulants in $B$. dorsalis and may attract the gravid females to lay more eggs $[17,18]$.
Therefore, we screened the female-biased chemosensory genes and compared the expression of these genes in the virgin and mated flies. We found that the expression of five candidate genes (SNMP 1, OBP 56d-1, OBP 56d-2, OR $7 \mathrm{a}-2$, and OR 43b-2) were upregulated after mating. The results indicated that these five genes may be involved in perception of volatiles from host plants for seeking oviposition site. Similar results have been observed in a study of Dendrolimus punctatus [31]. The previous study showed that chemosensory genes could be activated by a given odorant in vivo and that odorants could induce a fast change in the transcription level of activated chemosensory genes [32, 33]. In this study, odorant exposure assays showed that OBP $56 \mathrm{~d}-2$ was upregulated when the flies were exposed to benzothiazole. Furthermore, the fold-change of expression level in mated females was much higher than that in virgin females. Similar results were observed for three chemosensory genes (OBP 56d-1, OBP 56d-2, and OR 7a-1) in the 1-octen-3-ol exposure assay. This suggested that the mated flies were more sensitive to oviposition stimulants, which is entirely consistent with their biological characteristics. In addition, all these changed genes have higher expression levels after mating. Mated flies would rapid change their physiology and behavior, the gravid state influences their perception of odors and taste along with the increased feeding and egg laying behaviors [34, 35]. In addition, a previous study showed that 1-octen-3-ol and benzothiazole are more attractive to $B$. dorsalis after mating, and EAG responses are also stronger after mating [36]. Taken together, we speculated that OBP $56 \mathrm{~d}-2$ might be responsible for perception of benzothiazole, whereas OBP 56d-1, OBP 56d-2, OR 7a1 , and OR 43b-2 may be responsible for 1-octen-3-ol perception in $B$. dorsalis, which will facilitate the location of 
oviposition sites in this fly. Nevertheless, more solid evidence should be further pursued to confirm the relationship between these candidate chemosensory genes and the oviposition stimulants.

\section{Conclusion}

This study sequenced the antennal comparative transcriptome of $B$. dorsalis in different sexes and constructed DEG libraries. From the transcriptomic analysis, we screened 1151 DEGs, including 24 chemosensory genes. Among these differentially expressed chemosensory genes, there were 12 female-biased genes and five of them were upregulated after mating. The expression level of OBP 56d-2 increased after exposed to benzothiazole for $2 \mathrm{~h}$, and the expression level of OBP 56d-1, OBP 56d-2, OR 7a-1, and OR 43b-2 was upregulated after exposed to 1-octen-3-ol for $2 \mathrm{~h}$. The fold-change of expression level was higher in mated flies, indicating mated flies might be more sensitive to oviposition stimulants. In addition, OBP 56d-1, OBP 56d-2, OR 7a-1, and OR 43b-2 might be responsible for percepting oviposition stimulants and may be involved in locating oviposition sites. The results from this study have laid a solid foundation for the further research regarding the mechanism of benzothiazole-and 1-octen-3-ol-mediated oviposition behavior in $B$. dorsalis.

\section{Methods}

\section{Insects}

Oriental fruit flies were reared at the Key Laboratory of Entomology and Pest Control Engineering in Chongqing, China. The laboratory colony of $B$. dorsalis was originally collected from Haikou, Hainan province, China, in 2008. The insects were reared in the laboratory at $27 \pm 1{ }^{\circ} \mathrm{C}, 70 \pm 5 \%$ relative humidity, with a $14 \mathrm{~h}$ light: $10 \mathrm{~h}$ dark photoperiod [37]. Adults were fed an aqueous artificial diet of yeast powder, honey, sugar, ascorbic acid [38].

\section{RNA isolation, cDNA library preparation, and Illumina sequencing}

Samples were collected from five-day-old adult B. dorsalis and divided into two groups by sex. Each group had three independently biological replications and each replication contained antennae from 100 individuals. The antennae were dissected on the chilled ice and immediately frozen in liquid nitrogen.

Total RNA was extracted from the antennae of B. dorsalis using TRIzol reagent (Invitrogen Life Technologies, Carlsbad, CA) with DNase (Promega, Madison, WI) treatment to remove genomic DNA, followed by a phenol-chloroform extraction [39]. The purity of all RNA samples was assessed by absorbance ratios of $\mathrm{OD}_{260 / 280}$ and $\mathrm{OD}_{260 / 230}$, whereas the integrity of RNA was verified by electrophoresis on a 1.0\% agarose gel and the concentration of RNA was determined by Nanodrop One (Thermo Fisher Scientific, Madison, WI). The cDNA library construction for Illumina sequencing was conducted with $4 \mu \mathrm{g}$ RNA from each sample by using the Illumina TruSeq RNA Sample Preparation Kit by the manufacturer instructions. Sample sequencing was conducted on Illumina HiseqTM 2000 using paired-end technology with the PE150 sequencing strategy.

\section{De novo assembly and bioinformatics analysis}

The raw reads in the FASTQ format were cleaned by removing adapter sequences [40]. Short or low-quality reads containing $>5 \%$ unknown nt " $\mathrm{N}$ " and reads with $20 \%$ quality value $<10$ were removed from datasets to acquire more reliable results. The clean reads were de novo assembled into contiguous sequences (contigs) with the Trinity Method [41] to recover more full-length transcripts across a broad range of expression levels and sequencing depths with an optimized k-mer length of 25 for de novo assembly [41]. The assembled contigs were further clustered using TGI Clustering Tool [42] and the transcripts were clustered based on nucleotide sequence identity.

\section{The annotation and analysis of DEGs}

All raw sequence reads were filtered using the Illumina pipeline before mapping reads to the reference transcriptome database. Low-quality reads were removed from the data analysis. Low-quality reads include those which had more than $10 \%$ ambiguous bases and the quality value of those in more than $50 \%$ reads were $\leq 5$. The level of gene expression was determined by normalizing the number of unambiguous tags in each sample to RPKM [42]. The relative gene expression in male antenna or female antenna was equal to the mean of three biological samples from each group. The fold change was calculated as fold change with $\log _{2}$ ratio. The differentially expressed transcripts between samples were identified using a Bayesian algorithm [43]. The false discovery rate (FDR) method was used to determine the threshold of the $P$-value in multiple tests and analyses. Genes with an adjusted $P$-value $<0.05$ were for comparisions of gene expression and genes expressed at different levels across the samples were further annotated by $\mathrm{GO}$ function.

\section{Quantitative real-time PCR validation of the DEGs}

To verify the DEG results, the differentially expressed chemosensory genes were analyzed by qRT-PCR assay. Gene-specific primers and the target genes are listed into Table S1. The male and female antenna of five-dayold adult $B$. dorsalis were collected and each group contained 50 antennae with four biological replicates. RNA 
was extracted as described above. Total RNA $(1 \mu \mathrm{g})$ was treated with RNase-free DNase (Promega, Madison, WI, USA) to remove the genomic DNA at $37^{\circ} \mathrm{C}$ for $30 \mathrm{~min}$. First strand cDNA was synthesized with the PrimerScript RT Reagent kit (Takara, Dalian, China) following the manufacturer instructions.

The qRT-PCR was performed using CFX384TM RealTime System (Bio-Rad, Singapore, Jurong) with a $10 \mu \mathrm{L}$ reaction volume. The amplification efficiency of each primer was first determined with a standard curve based on a 5 -fold cDNA dilution series. Then, the expression profile in male and female antennae was performed using the appropriate primers. Each $10 \mu \mathrm{L}$ reaction mixture contain $5 \mu \mathrm{L}$ SYBR supermix (Novoprotein, Shanghai, China), $3.9 \mu \mathrm{L}$ nuclease-free water, $0.5 \mu \mathrm{L}$ of each cDNA sample (approximately $300 \mathrm{ng} / \mu \mathrm{L}$ ), and $0.3 \mu \mathrm{L}$ forward and reverse primers $(10 \mu \mathrm{M})$. The procedure for PCR was as follows: an initial denaturation at $95^{\circ} \mathrm{C}$ for $2 \mathrm{~min}$, followed by 40 cycles at $95^{\circ} \mathrm{C}$ for $15 \mathrm{~s}$, and incubation at $60^{\circ} \mathrm{C}$ for $30 \mathrm{~s}$. A melt curve was performed to ensure the specificity from 60 to $95^{\circ} \mathrm{C}$ [39]. The double internal reference genes $\alpha$-tubulin (GenBank: GU269902) and ribosomal protein S3 (rps 3, GenBank: XM_011212815) were applied because of their stable expression level in $B$. dorsalis. The relative expression was calculated with Biogazelle qBase software and the data were analyzed with SPSS 16.0 using one-way analysis of variance (ANOVA) followed by Turkey's honestly significantly difference test $(P<0.05)$.

\section{Tissues and odorant-induced expression profiling}

Different tissues including antenna, proboscis, maxillary palps, head cuticles, thorax cuticles, abdomen cuticles, legs, and wings were dissected from female flies. In addition, the head of 15-day-old virgin and mated female flies were dissected separately. The total RNA of these tissues was extracted for four biological replicates using the method as mentioned above. To detect the expression profile of the female-biased genes after the benzothiazole and 1-octen-3-ol exposure, a bioassay was conducted using $5 \%(\mathrm{vol} / \mathrm{vol})$ benzothiazole $(\geq 96 \%$, sigma, USA) and 1-octen-3-ol ( $\geq 98 \%$, sigma, USA). The odorants were diluted in mineral oil (MO, sigma, USA) whereas the MO-only served as the control. Odorants were applied to 15-day-old virgin and mated female flies. The assay was performed at a $700 \mathrm{~mL}$ transparent cage in a $27^{\circ} \mathrm{C}$ incubator. Filter strips $(5 \times 1 \mathrm{~cm})$ loaded with $50 \mu \mathrm{L} 5 \%$ benzothiazole, 1-octen-3-ol, or MO were placed at the center of the cage. Each cage contained 50 female flies. Before odorant exposure, the flies were starved for $8 \mathrm{~h}$. Exposure started in the morning at $9 \mathrm{am}$ and lasted for $2 \mathrm{~h}$. The exposure of different odorants was conducted in different incubators with three replicates. After the exposure, antennae of flies were dissected on ice and prepared for tissue extraction. The total RNA and first strand cDNA were obtained as described above. The tissues and odorant-induced expression profilings were detected by qPCR.

\section{Supplementary Information}

The online version contains supplementary material available at https://doi. org/10.1186/s12864-020-07325-z.

Additional file 1: Figure S1. Homology analysis of unigenes in $B$. dorsalis for species distribution. The species distribution was shown as percentage of total homologous sequences in the NCBI NR protein database. The different colors represented different species.

Additional file 2: Figure S2. Venn diagram for four databases: NR, KEGG, Swissport, and KOG.

Additional file 3: Figure S3. Heat map of correlation between samples. A relative color scheme used to represent the correlation coefficient between samples. MAn represents male antenna, FAn represents female antenna.

Additional file 4: Figure S4. Pearson's correlation of gene expression fold changes from female flies ( $\log _{2}$ fold change) measured using RNA sequencing (RNA-seq) and real-time quantitative PCR (qPCR). MAn represents male antenna, FAn represents female antenna.

Additional file 5: Table S1. Primer sequences of candidate olfactory genes used for qRT-PCR.

\section{Abbreviations}

RNA-seq: RNA sequencing; DEGs: Differentially expressed genes;

OSNs: Olfactory sensory neurons; OBPs: Odorant binding proteins;

SNMP: Sensory neuron membrane proteins; CSPs: Chemosensory proteins;

ODEs: Odorant degrading enzymes; ORs: Odorant receptors; GRs: Gustatory receptors; IRs: Ionotropic receptors; ME: Methyl eugenol; RPKM: Reads per kilobase mapped; FDR: False discovery rate; MO: Mineral oil

\section{Acknowledgments}

We thank LetPub (www.letpub.com) for its linguistic assistance during the preparation of this manuscript.

\section{Authors' contributions}

$\mathrm{H}-\mathrm{BJ}$ and J-JW designed the research and provided the materials and reagents. LX, K-YT, X-FC, and $Y T$ performed all of the experiments. $L X$ designed primers for qRT-PCR. YT reared the test insects. $L X$, and $H-B J$ analyzed the data. LX wrote the original draft. H-BJ and J-JW edited and modified the manuscript. All authors have read and approved the manuscript.

\section{Funding}

This study was supported in part by the National Natural Science Foundation of China (31772233), Fok Ying Tung Education Foundation (161029), Chongqing Natural Science Foundation (cstc2019jcyj-zdxmX0034),

Chongqing Young Talents Support Program, and the earmarked fund for Modern Agro-Industry (Citrus) Technology Research System (CARS-26) of China.

\section{Availability of data and materials}

All datasets for this study are included in the manuscript and/or the supplementary files. All row reads of the transcriptome had been archived at the Short Read Archive (SRA) in NCBI under project accession number PRJNA657376 and biosample accession numbers SRR12473599-SRR12473604.

Ethics approval and consent to participate Not applicable.

Consent for publication

Not applicable.

Competing interests

The authors declare no compete interests. 
Received: 4 September 2020 Accepted: 14 December 2020 Published online: 06 January 2021

\section{References}

1. Zhao S, Xing Z, Liu Z, Liu Y, Liu X, Chen Z, Li J, Yan R. Efficient somatic and germline genome engineering of Bactrocera dorsalis by the CRISPR/Cas9 system. Pest Manag Sci. 2019;75(7):1921-32.

2. Ohno S, Tamura Y, Haraguchi D, Matsuyama T, Kohama T. Re-invasions by Bactrocera dorsalis complex (Diptera: Tepritidae) occurred after its eradication in Okinawa, Japan, and local differences found in the frequency and temporal patterns of invasions. Appl Entomol Zool. 2009;44(4):643-54.

3. Lu XP, Wang LL, Huang Y, Dou W, Chen CT, Wei D, Wang JJ. The epsilon glutathione S-transferases contribute to the malathion resistance in the oriental fruit fly, Bactrocera dorsalis (Hendel). Comp Biochem Physiol. 2016; 180:40-8.

4. Jin T, Zeng L, Lin YY, Lu YY, Liang GW. Insecticide resistance of the oriental fruit fly, Bactrocera dorsalis (Hendel) (Diptera: Tephritidae), in mainland China. Pest Manag Sci. 2011;67(3):370-6.

5. Haney S, Saha D, Raman B, Bazhenov M. Differential effects of adaptation on odor discrimination. J Neurophysiol. 2018;120(1):171-85.

6. Leal WS. Odorant reception in insects: roles of receptors, binding proteins, and degrading enzymes. Annu Rev Entomol. 2013;58:373-91.

7. Yuvaraj JK, Andersson MN, Anderbrant O, Lofstedt C. Diversity of olfactory structures: a comparative study of antennal sensilla in Trichoptera and Lepidoptera. Micron. 2018;111:9-18.

8. Suh E, Bohbot JD, Zwiebel LJ. Peripheral olfactory signaling in insects. Curr Opin Insect Sci. 2014;6:86-92.

9. Liu Y, Gu S, Zhang Y, Guo Y, Wang G. Candidate olfaction genes identified within the Helicoverpa armigera antennal transcriptome. PLoS One. 2012; 7(10):e48260.

10. Cao D, Liu Y, Wei J, Liao X, Walker WB, Li J, Wang G. Identification of candidate olfactory genes in Chilo suppressalis by antennal transcriptome analysis. Int J Biol Sci. 2014;10(8):846-60.

11. Liu Y, Du L, Zhu Y, Yang S, Zhou Q, Wang G, Liu Y. Identification and sexbiased profiles of candidate olfactory genes in the antennal transcriptome of the parasitoid wasp Cotesia vestalis. Comp Biochem Physiol. 2020;34: 100657

12. Yang B, Ozaki K, Ishikawa Y, Matsuo T. Identification of candidate odorant receptors in Asian corn borer Ostrinia furnacalis. PLoS One. 2015;10(3): e0121261.

13. Bachmann GE, Segura DF, Devescovi F, Juarez ML, Ruiz MJ, Vera MT, Cladera $J$, Teal PEA, Fernandez PC. Male sexual behavior and pheromone emission is enhanced by exposure to guava fruit volatiles in Anastrepha fraterculus. PLoS One. 2015;10(4):e0124250.

14. Steiner LF. Methyl eugenol as an attractant for oriental fruit fly. J Econ Entomol. 1952:45(2):241-8.

15. Liu H, Zhao X-F, Fu L, Han Y-Y, Chen J, Lu Y-Y. BdorOBP2 plays an indispensable role in the perception of methyl eugenol by mature males of Bactrocera dorsalis (Hendel). Sci Rep. 2017;7:1-4.

16. Liu H, Chen Z-S, Zhang D-J, Lu Y-Y. BdorOR88a modulates the responsiveness to methyl eugenol in mature males of Bactrocera dorsalis (Hendel). Front Physiol. 2018:9:987.

17. Jayanthi PDK, Kempraj V, Aurade RM, Venkataramanappa RK, Nandagopal B, Verghese A, Bruce TJA. Specific volatile compounds from mango elicit oviposition in gravid Bactrocera dorsalis females. J Chem Ecol. 2014;40(3): 259-66.

18. Kamala Jayanthi PD, Kempraj V, Aurade RM, Bruce TJA. Evaluation of synthetic oviposition stimulants to enhance egg collection of the oriental fruit fly, Bactrocera dorsalis (Diptera: Tephritidae). J Pest Sci. 2017:90(3):781-6.

19. Liu Z, Smagghe G, Lei Z, Wang J-J. Identification of male- and femalespecific olfaction genes in antennae of the Oriental Fruit Fly (Bactrocera dorsalis). Plos One. 2016;11(2):e0147783.

20. Wang $Y$, Jiang $H$, Yang L. Transcriptome analysis of Zebrafish olfactory epithelium reveal sexual differences in odorant detection. Genes. 2020;11(6): 592

21. Bin SY, Qu MQ, Pu XH, Wu ZZ, Lin JT. Antennal transcriptome and expression analyses of olfactory genes in the sweetpotato weevil Cylas formicarius. Sci Rep. 2017;7(1):11073.

22. Wang $H$, Zheng $H$, Zhang $Y$, Zhang $X$. Morphology and distribution of antennal, maxillary palp and labial palp sensilla of the adult bruchid beetles,
Callosobruchus chinensis(L.) (Coleoptera: Bruchidae). Entomol Res. 2018;48(6): 466-79.

23. Ahn S-J, Oh H-W, Corcoran J, Kim J-A, Park K-C, Park CG, Choi M-Y. Sexbiased gene expression in antennae of Drosophila suzukii. Arch Insect Biochem Physiol. 2020;104(1):e21660.

24. Yang P, Chen X-M, Liu W-W, Feng Y, Sun T. Transcriptome analysis of sexually dimorphic Chinese white wax scale insects reveals key differences in developmental programs and transcription factor expression. Sci Rep. 2015;5:1-8.

25. Li RT, Huang LQ, Dong JF, Wang CZ. A moth odorant receptor highly expressed in the ovipositor is involved in detecting host-plant volatiles. Elife. 2020;9:e53706

26. Segura DF, Belliard SA, Vera MT, Bachmann GE, Ruiz MJ, Jofre-Barud F, Fernandez PC, Lopez ML, Shelly TE. Plant chemicals and the sexual behavior of male Tephritid fruit flies. Ann Entomol Soc Am. 2018;111(5):239-64.

27. Day JF. Mosquito oviposition behavior and vector control. Insects. 2016;7(4):65.

28. Krieger J, Grosse-Wilde E, Gohl T, Dewer YME, Raming K, Breer H. Genes encoding candidate pheromone receptors in a moth (Heliothis virescens). Proc Natl Acad Sci U S A. 2004;101(32):11845-50.

29. Zhou J, Zhang N, Wang P, Zhang SC, Li DQ, Liu KY, Wang GX, Wang XP, Ai $\mathrm{H}$. Identification of host-plant volatiles and characterization of two novel general odorant-binding proteins from the legume pod borer, Maruca vitrata Fabricius (Lepidoptera: Crambidae). PLoS One. 2015:10(10):e0141208.

30. Zhang J, Luo D, Wu P, Li H, Zhang H, Zheng W. Identification and expression profiles of novel odorant binding proteins and functional analysis of OBP99a in Bactrocera dorsalis. Arch Insect Biochem Physiol. 2018; 98(1):e21452.

31. Zhang SF, Zhang Z, Kong XB, Wang HB, Liu F. Dynamic changes in chemosensory gene expression during the Dendrolimus punctatus mating process. Front Physiol. 2018:8:1127.

32. von der Weid B, Rossier D, Lindup M, Tuberosa J, Widmer A, Dal Col J, Kan C, Carleton A, Rodriguez I. Large-scale transcriptional profiling of chemosensory neurons identifies receptor-ligand pairs in vivo. Nat Neurosci. 2015;18(10):1455.

33. Yin J, Wang C, Fang C, Zhang S, Cao Y, Li K, Leal WS. Functional characterization of odorant-binding proteins from the scarab beetle Holotrichia oblita based on semiochemical-induced expression alteration and gene silencing. Insect Biochem Mol Biol. 2019:104:11-9.

34. Hussain A, Ucpunar HK, Zhang M, Loschek LF, Grunwald Kadow IC. Neuropeptides modulate female chemosensory processing upon mating in Drosophila. PLoS Biol. 2016;14(5):e1002455.

35. Krupp JJ, Levine JD. Neural circuits: anatomy of a sexual behavior. Curr Biol. 2014:24(8):R327-9.

36. Wu Z, Lin J, Zhang H, Zeng X. BdorOBP83a-2 mediates responses of the oriental fruit fly to semiochemicals. Front Physiol. 2016;7:452.

37. Hou QL, Chen EH, Jiang HB, Wei DD, Gui S-H, Wang J-J, Smagghe G. Adipokinetic hormone receptor gene identification and its role in triacylglycerol mobilization and sexual behavior in the oriental fruit fly (Bactrocera dorsalis). Insect Biochem Mol Biol. 2017:90:1-13.

38. Shen GM, Dou W, Huang Y, Jiang XZ, Smagghe G, Wang JJ. In silico cloning and annotation of genes involved in the digestion, detoxification and RNA interference mechanism in the midgut of Bactrocera dorsalis Hendel (Diptera: Tephritidae). Insect Mol Biol. 2013;22(4):354-65.

39. Xu L, Jiang HB, Chen XF, Xiong Y, Lu XP, Pei YX, Smagghe G, Wang JJ. How tyramine beta-hydroxylase controls the production of octopamine, modulating the mobility of beetles. Int J Mol Sci. 2018;19(3):846.

40. Shang F, Ding B-Y, Xiong Y, Dou W, Wei D, Jiang HB, Wei DD, Wang JJ. Differential expression of genes in the alate and apterous morphs of the brown citrus aphid, Toxoptera citricida. Sci Rep. 2016;6:32099.

41. Grabherr MG, Haas BJ, Yassour M, Levin JZ, Thompson DA, Amit I, Adiconis X, Fan $L$, Raychowdhury R, Zeng $Q$, et al. Full-length transcriptome assembly from RNASeq data without a reference genome. Nat Biotechnol. 2011;29(7):644-U130.

42. Pertea G, Huang XQ, Liang F, Antonescu V, Sultana R, Karamycheva S, Lee $Y$, White J, Cheung F, Parvizi B, et al. TIGR gene indices clustering tools (TGICL): a software system for fast clustering of large EST datasets. Bioinformatics. 2003;19(5):651-2.

43. Audic S, Claverie JM. The significance of digital gene expression profiles. Genome Res. 1997;7(10):986-95.

\section{Publisher's Note}

Springer Nature remains neutral with regard to jurisdictional claims in published maps and institutional affiliations. 